\title{
Parameter identification of weakly nonlinear vibration system in frequency domain
}

\author{
Jiehua Peng ${ }^{\mathrm{a}, \mathrm{b}, *}$, Jiashi Tang ${ }^{\mathrm{a}}$ and Zili Chen ${ }^{\mathrm{a}, \mathrm{c}}$ \\ ${ }^{a}$ Department of Mechanics, Hunan University, Changsha, 410082, P.R. China \\ ${ }^{\mathrm{b}}$ Department of physics, Shaoyang University, Shaoyang, 422000, P.R. China \\ ${ }^{\mathrm{c}}$ College of Architectural Engineering, Central South Forestry University, Zhuzhou, 412008, P.R. China
}

Received 1 November 2001

Revised 11 December 2003

\begin{abstract}
A new method of identifying parameters of nonlinearly vibrating system in frequency domain is presented in this paper. The problems of parameter identification of the nonlinear dynamic system with nonlinear elastic force or nonlinear damping force are discussed. In the method, the mathematic model of parameter identification is frequency response function. Firstly, by means of perturbation method the frequency response function of weakly nonlinear vibration system is derived. Next, a parameter transformation is made and the frequency response function becomes a linear function of the new parameters. Then, based on this function and with the least square method, physical parameters of the system are identified. Finally, the applicability of the proposed technique is confirmed by numerical simulation.
\end{abstract}

Keywords: Parameter identification, weakly nonlinear vibration system, least square method, frequency domain

\section{Introduction}

The parameter identification is one of the important problems of the vibration research. There are many methods of identifying parameters of the linear system. But the parameter identification of nonlinear system is much more difficult than the linear system. Hence, this problem attracts much attention. A. H. Nayfeh has presented an excellent method of parametric identification of nonlinear system that exploits nonlinear resonances and comparisons of the system to be identified with those of known system [1], but the difficult usually appears when we find the known system. Tang et al. [2-4] have suggested time domain method and phase plane method for identifying parameters of nonlinear system which are effective for integrable nonlinear system. K. Yasuda et al. [5,6] have proposed an experiment method of parameter identification which is useful for geometrically nonlinear systems. R. Bachmayer et al. [7] have established adaptive parameter identification method for marine thruster which is an on-line technique for adaptive identification. There are some other methods of parameter identification, such as orthogonal function method [8], wavelet-based technique [9], Volterra series method [10], cross-correlation analysis [11], power spectrum technique [12], and so on.

A new method of identifying parameters of the weakly nonlinear vibration system in the frequency domain is presented in this paper. We discuss two kinds of nonlinear vibration systems with nonlinear elastic force or nonlinear damping force respectively. First, by using the multiple scale method, we obtain the frequency response function of the system which is regarded as a mathematical model of the curve fitting. Next, a new set of parameters are introduced to replace the parameters to be identified and the frequency response function is transformed into the linear function of the new parameters so that the least square method can be used to identify the parameters. This

*Corresponding author. E-mail: Pengjhua @ sina.com. 
is the key step of our method. Then, with the least square method the normal equations are established and they are linear equations of the new parameters. From the solution of normal equations we can get the values of the parameters to be identified.

The method we present is a combination of the perturbation method and the least square method, hence, the method has the same precision with those of perturbation method and least square method. On the other hand, the perturbation method is an effective method to deal with weakly nonlinear system and the least square method is an excellent method of curve fitting such that this parameter identification method has a wide application in the study of weakly nonlinear vibration system. Although frequency response function is the first order approximation of the relationship between amplitude and frequency of nonlinear system, it is the most useful and important mathematical model to study the property of nonlinear vibration system [13]. It can be determined by experiment with good accuracy because in practice any complicated nonlinear system will finally move in a steady motion state if the excitation is harmonic and the system doesn't come into chaotic motion. Compared with response function in time domain, frequency response function is independent of initial conditions and the test to determine this relationship possesses good repeatability and stability, such that this method of parameter identification can be easily realized by experiment.

\section{Principle of the method}

The governing equation of the vibration system with nonlinear damping force is as follows

$$
m \ddot{x}+c \dot{x}+f(\dot{x})+k x=G \cos \Omega t
$$

The governing equation of the vibration system with nonlinear elastic force is as follows

$$
m \ddot{x}+c \dot{x}+k x+f(x)=G \cos \Omega t
$$

where $f(\dot{x})$ is a nonlinear function of $\dot{x}, f(x)$ is a nonlinear function of $x$. Rewrite Eqs (1) and (2) into the standard form

$$
\ddot{x}+\omega^{2} x=\varepsilon\left[-2 n \dot{x}-f_{1}(\dot{x})+g \cos \Omega t\right]
$$

and

$$
\ddot{x}+\omega^{2} x=\varepsilon\left[-2 n \dot{x}-f_{1}(x)+g \cos \Omega t\right]
$$

where $\varepsilon$ being a small positive parameter, $\omega=\frac{k}{m}$ being the linear natural frequency of the system, $n=\frac{c}{2 m}$, $f_{1}(\dot{x})=\frac{f(\dot{x})}{m}, f_{1}(x)=\frac{f(x)}{m}$ and $g=\frac{G}{m}$. When $\Omega$ approaches to $\omega$, we introduce a detuning parameter $\sigma$, letting

$$
\Omega=\omega+\varepsilon \sigma
$$

We can find the approximate frequency response function of the nonlinear system Eqs (3) and (4) by using approximate analytical method, such as the methods of iteration, multiple scales, KBM and so on [13-15].

The general approximate frequency response function of Eqs (3) and (4) can be written in the form

$$
F(\omega, n, \ldots, a, \sigma)=0
$$

Where $\omega, n, \ldots$, are physical parameters to be identified of the system Eqs (3) and (4), $a$ is the amplitude, $\sigma$ is the detuning parameter whose dimension is the same with frequency.

Equation (6) plays key role in revealing the properties of nonlinear vibration system and here we take it as a mathematical model of the curve fitting. But $F(\omega, n, \ldots, a, \sigma)$ is a nonlinear function of parameters $(\omega, n, \ldots$, we must replace the parameters $(\omega, n, \ldots$,$) with a new set of parameters (A, B, \ldots$,$) before employing the least$ square method to identify the parameters, such that $F(\omega, n, \ldots, a, \sigma)$ can be transformed into a linear function of $(A, B, \ldots$,$) . That is, if we chose$

$$
(\omega, n, \ldots,)=R(A, B, \ldots,)
$$

then 


$$
F(\omega, n, \ldots, a, \sigma)=F_{1}(A, B, \ldots, a, \sigma)=0
$$

Where $F_{1}(A, B, \ldots, a, \sigma)$ is a linear function of parameters $(A, B, \ldots$,$) . Transform function R(A, B, \ldots$,$) is$ determined by the frequency response function of the systems. We use the test data $\left(\sigma_{i}, a_{i}\right)(i=1,2, \ldots, m)$ for fitting the curve. Substituting $\sigma_{i}, a_{i}$ into Eq. (8) yields small errors

$$
E_{i}=F_{1}\left(A, B, \ldots, a_{i}, \sigma_{i}\right) i=1,2, \ldots, m
$$

The sum of the square of all errors is

$$
E=\sum_{i=1}^{m} E_{i}^{2}=\sum_{i=1}^{m}\left[F_{1}\left(A, B, \ldots, a_{i}, \sigma_{i}\right)\right]^{2}
$$

It is obvious that the parameters $\omega, n \ldots$ will fit to make the totle square errors $E$ get the minimum. This means that we can use the method of the minimum squares to identify the parameters of the system. By determining the extreme value of $E$ and solving a set of normal equations resulted from the extreme value of $E$, we can identify parameters $\omega, n, \ldots$ of the nonlinear system.

\section{The system with nonlinear damping}

Let us consider a weakly nonlinear vibration system with a small cubic nonlinear damping force, its governing equation is as follows.

$$
m \ddot{x}+c \dot{x}+c_{1} \dot{x}^{3}+k x=G \cos \Omega t
$$

Rewrite the standard equation in the form

$$
m \ddot{x}+\omega^{2} x=\varepsilon\left(-2 n \dot{x}-\alpha \dot{x}^{3}+g \cos \Omega t\right)
$$

where

$$
\omega^{2}=\frac{k}{m}, 2 n=\frac{g}{m}, \quad \alpha=\frac{c}{m}, g=\frac{G}{m}
$$

$\omega, n$ and $\alpha$ are the physical parameters to be identified of the system Eq. (11). We begin by assuming an expansion of the solution having the form

$$
x(t, \varepsilon)=x_{0}\left(T_{0}, T_{1}\right)+\varepsilon x_{1}\left(T_{0}, T_{1}\right)+\ldots
$$

New independent variables are introduced according to

$$
T_{n}=\varepsilon^{n} t \text { for } n=0,1, \ldots
$$

Substituting Eq. (13) into Eq. (11) and equating the coefficients of $\varepsilon^{i},(i=0,1,2, \ldots)$ to zero, we obtain

$$
\begin{aligned}
& D_{0}^{2} x_{0}+\omega^{2} x_{0}=0 \\
& D_{0}^{2} x_{1}+\omega^{2} x_{1}=-2 D_{0} D_{1} x_{0}-2 n D_{0} x_{0}-\alpha\left(D_{0} x_{0}\right)^{3}+g \cos \left(\omega T_{0}+\sigma T_{1}\right)
\end{aligned}
$$

The solution of the Eq. (15) is

$$
x_{0}=a \cos \left(\omega T_{0}+\varphi\right)
$$

Substituting Eq. (17) into Eq. (16) leads to

$$
\begin{aligned}
D_{0}^{2} x_{1}+\omega^{2} x_{1}= & 2 \omega a^{\prime} \sin \left(\omega T_{0}+\varphi\right)+2 \omega a \beta^{\prime} \cos \left(\omega T_{0}+\varphi\right)+2 n \omega a \sin \left(\omega T_{0}+\varphi\right)+\frac{1}{4} \alpha a^{3} \omega^{3}\left[3 \sin \left(\omega T_{0}+\varphi\right)\right. \\
& \left.-\sin 3\left(\omega T_{0}+\varphi\right)\right]+g \cos \left(\omega T_{0}+\varphi\right) \cos \left(\sigma T_{1}-\varphi\right)-g \sin \left(\omega T_{0}+\varphi\right) \sin \left(\sigma T_{1}-\varphi\right)
\end{aligned}
$$

To eliminate secular terms from $x_{1}$, we must put 


$$
\left\{\begin{array}{l}
2 \omega a^{\prime}+2 n a \omega+\frac{3}{4} \alpha a^{3} \omega^{3} \\
-g \sin \left(\sigma T_{1}-\varphi\right)=0 \\
2 \omega a \varphi^{\prime}+g \cos \left(\sigma T_{1}-\varphi\right)=0
\end{array}\right.
$$

Letting

$$
\sigma T_{1}-\varphi=\gamma
$$

Determining the derivatives with respect to $T_{1}$ yields $\varphi^{\prime}=\sigma-\gamma^{\prime}$. Considering the steady-state response, that is, $a^{\prime}=0, \gamma^{\prime}=0$, we have

$$
\left\{\begin{array}{l}
2 n a \omega+\frac{3}{4} \alpha a^{3} \omega^{3}-g \sin \gamma=0 \\
2 \omega a \sigma+g \cos \gamma=0
\end{array}\right.
$$

The result by eliminating $\gamma$ in Eq. (20) is

$$
4 n^{2} \omega^{2} a^{2}+3 \alpha n \omega^{4} a^{4}+\frac{9}{16} \alpha^{2} \omega^{6} a^{6}+4 \omega^{2} \sigma^{2} a^{2}-g^{2}=0
$$

This is the first-order approximation frequency response function of the weakly nonlinear system Eq. (11). For identifying physical parameters $\omega, n$ and $\alpha$ of the system with test data $\left\{\sigma_{i}, a_{i}\right\}(i=1,2, \ldots, m)$, Following transformation should be introduced

$$
A=4 n^{2} \omega^{2}, \quad B=3 \alpha n \omega^{4}, \quad C=\frac{9}{16} \alpha^{2} \omega^{6}, \quad D=4 \omega^{2}
$$

therefore Eq. (21) becomes

$$
A a^{2}+B a^{4}+C a^{6}+D \sigma^{2} a^{2}-g^{2}=0
$$

Equation (23) is a frequency response equation of the nonlinear system Eq. (11). We regard it as a mathematical model of the parameter identification. Setting the testing values of $(\sigma, a)$ are $\left\{\sigma_{i}, a_{i}\right\}(i=1,2 \ldots, m)$ and substituting them into Eq. (23) generating errors as follow

$$
E_{i}=a_{i}^{2} A+a_{i}^{4} B+a_{i}^{6} C+\sigma_{i}^{2} a_{i}^{2} D-g^{2} i=1,2 \ldots, m
$$

The sum of the square of all errors is

$$
E=\sum_{i=1}^{m}\left(a_{i}^{2} A+a_{i}^{4} B+a_{i}^{6} C+\sigma_{i}^{2} a_{i}^{2} D-g^{2}\right)^{2}
$$

The best way to determine $A, B, C$ and $D$ is choosing those values of them that make the sum of the square of all errors $E$ be minimum. This means that the difference between the theoretical result and the experiment result is least. Noting that the error $E$ is the function of $A, B, C$ and $D$, the necessary conditions of $E$ getting minimum are that the partial derivatives of $E$ with respect to $A, B, C$ and $D$ equal zero, respectively, that is,

$$
\frac{\partial E}{\partial A}=0, \frac{\partial E}{\partial B}=0, \frac{\partial E}{\partial C}=0, \frac{\partial E}{\partial D}=0
$$

namely,

$$
\begin{aligned}
& \sum_{i=1}^{m}\left(a_{i}^{2} A+a_{i}^{4} B+a_{i}^{6} C+\sigma_{i}^{2} a_{i}^{2} D-g^{2}\right) a_{i}^{2}=0 \\
& \sum_{i=1}^{m}\left(a_{i}^{2} A+a_{i}^{4} B+a_{i}^{6} C+\sigma_{i}^{2} a_{i}^{2} D-g^{2}\right) a_{i}^{4}=0 \\
& \sum_{i=1}^{m}\left(a_{i}^{2} A+a_{i}^{4} B+a_{i}^{6} C+\sigma_{i}^{2} a_{i}^{2} D-g^{2}\right) a_{i}^{6}=0 \\
& \sum_{i=1}^{m}\left(a_{i}^{2} A+a_{i}^{4} B+a_{i}^{6} C+\sigma_{i}^{2} a_{i}^{2} D-g^{2}\right) \sigma_{i}^{2} a_{i}^{2}=0
\end{aligned}
$$


These equations are usually called normal equations in numerical analysis. They are linear algebraic equations of parameters $A, B, C, D$. According the theory of numerical analysis, the solution of the normal equations is sole and it makes the errors $\mathrm{E}$ get minimum definitively [16]. For the simplification we write the normal equations in the matrix form,

$$
Q \vec{y}=\vec{s}
$$

where

$$
\begin{aligned}
\vec{y} & =\left(\begin{array}{llll}
A & B & C & D
\end{array}\right)^{T} \\
\vec{s} & =\left(\begin{array}{llll}
g^{2} \sum_{i=1}^{m} a_{i}^{2} & g^{2} \sum_{i=1}^{m} a_{i}^{4} & g^{2} \sum_{i=1}^{m} a_{i}^{6} & g^{2} \sum_{i=1}^{m} \sigma_{i}^{2} a_{i}^{2}
\end{array}\right)^{T}
\end{aligned}
$$

and

$$
Q=\left[\begin{array}{cccc}
\sum_{i=1}^{m} a_{i}^{4} & \sum_{i=1}^{m} a_{i}^{6} & \sum_{i=1}^{m} a_{i}^{8} & \sum_{i=1}^{m} \sigma_{i}^{2} a_{i}^{4} \\
\sum_{i=1}^{m} a_{i}^{6} & \sum_{i=1}^{m} a_{i}^{8} & \sum_{i=1}^{m} a_{i}^{10} & \sum_{i=1}^{m} \sigma_{i}^{2} a_{i}^{6} \\
\sum_{i=1}^{m} a_{i}^{8} & \sum_{i=1}^{m} a_{i}^{10} & \sum_{i=1}^{m} a_{i}^{12} & \sum_{i=1}^{m} \sigma_{i}^{2} a_{i}^{8} \\
\sum_{i=1}^{m} \sigma_{i}^{2} a_{i}^{2} & \sum_{i=1}^{m} \sigma_{i}^{2} a_{i}^{6} & \sum_{i=1}^{m} \sigma_{i}^{2} a_{i}^{6} & \sum_{i=1}^{m} \sigma_{i}^{4} a_{i}^{4}
\end{array}\right]
$$

As mentioned above, the solution of normal equation is sole and this means matrix $Q$ is reversible. Solving matrix Eq. (25) yields $\vec{y}=Q^{-1} \vec{s}$. Substituting the values of $A, B, C$ and $D$ into Eq. (22) we obtain physical parameters of the nonlinear system in the form

$$
\omega=\frac{\sqrt{D}}{2}, \quad n=\sqrt{\frac{A}{D}}, \quad \alpha=\frac{B}{2 n \omega^{4}} \text { or } \alpha=\frac{4 \sqrt{C}}{3 \omega^{3}}
$$

\section{The system with cubic elastic force}

In the case of a nonlinear vibration system with a small cubic nonlinear elastic force and linear viscous damping, the governing equation of it is

$$
m \ddot{x}+c \dot{x}+k x+k_{1} x^{3}=G \cos \Omega t
$$

Equation (28) can be rewritten as

$$
\begin{aligned}
& \ddot{x}+\omega^{2} x=\varepsilon\left(-2 n \dot{x}-\beta x^{3}+g \cos \Omega t\right) \\
& \omega^{2}=\frac{k}{m}, \quad 2 n=\frac{c}{m}, \quad \beta=\frac{k_{1}}{m}, \quad g=\frac{G}{m} .
\end{aligned}
$$

Using the method of multiple scales or KBM, we can derive the frequency response equation of Eq. (29) as follows

$$
4 n^{2} \omega^{2} a^{2}+4 \omega^{2} \sigma^{2} a^{2}-3 \beta \omega \sigma a^{4}+\frac{9}{16} \beta^{2} a^{6}-g^{2}=0
$$

Similarly, $A, B, C$ and $D$ are introduced, Eq. (31) becomes

$$
A a^{2}+B \sigma^{2} a^{2}+C \sigma a^{4}+D a^{6}-g^{2}=0
$$

Where 


$$
A=4 n^{2} \omega^{2}, \quad B=4 \omega^{2}, \quad C=-3 \beta \omega, D=\frac{9}{16} \beta^{2} .
$$

The sum of the square of all error arising in the procedure of substituting the testing data $\left\{\sigma_{i}, a_{i}\right\}(i=1,2, \ldots, m)$ into Eq. (32) is

$$
E=\sum_{i=1}^{m}\left(a_{i}^{2} A+\sigma_{i}^{2} a_{i}^{2} B+\sigma_{i} a_{i}^{4} C+a_{i}^{6} D-g^{2}\right)^{2}
$$

The normal equation corresponding to the minimum of error function $E$ can be obtained as follows

$$
\left[\begin{array}{llll}
\sum_{i=1}^{m} a_{i}^{4} & \sum_{i=1}^{m} \sigma_{i}^{2} a_{i}^{4} & \sum_{i=1}^{m} \sigma_{i} a_{i}^{6} & \sum_{i=1}^{m} a_{i}^{8} \\
\sum_{i=1}^{m} \sigma_{i}^{2} a_{i}^{4} & \sum_{i=1}^{m} \sigma_{i}^{4} a_{i}^{4} & \sum_{i=1}^{m} \sigma_{i}^{3} a_{i}^{6} & \sum_{i=1}^{m} \sigma_{i}^{2} a_{i}^{8} \\
\sum_{i=1}^{m} \sigma_{i} a_{i}^{6} & \sum_{i=1}^{m} \sigma_{i}^{3} a_{i}^{6} & \sum_{i=1}^{m} \sigma_{i}^{2} a_{i}^{8} & \sum_{i=1}^{m} \sigma_{i} a_{i}^{10} \\
\sum_{i=1}^{m} a_{i}^{8} & \sum_{i=1}^{m} \sigma_{i}^{2} a_{i}^{8} & \sum_{i=1}^{m} \sigma_{i} a_{i}^{10} & \sum_{i=1}^{m} a_{i}^{12}
\end{array}\right]\left[\begin{array}{l}
A \\
B \\
C \\
D
\end{array}\right]=\left[\begin{array}{l}
g^{2} \sum_{i=1}^{m} a_{i}^{2} \\
g^{2} \sum_{i=1}^{m} \sigma_{i}^{2} a_{i}^{2} \\
g^{2} \sum_{i=1}^{m} \sigma_{i} a_{i}^{4} \\
g^{2} \sum_{i=1}^{m} a^{6}
\end{array}\right]
$$

Solving Eq. (35) we obtain the values of $A, B, C$ and $D$.

Therefore physical parameters of the nonlinear system Eq. (29) are

$$
\omega=\frac{\sqrt{B}}{2}, n=\sqrt{\frac{A}{B}}, \beta=-\frac{C}{3 \omega}, \text { or, } \beta=\frac{4}{3} \sqrt{D}
$$

\section{Example and discussion}

With the method of numerical simulation we can check up the precision of the method. We take Eq. (29) for example, letting

$$
\omega=5, \quad n=0.1 \beta=0.4 g=9.8
$$

By substituting them into Eq. (29) and employing multiple scales method, we get the frequency response equation as follows

$$
a^{2}+100 \sigma^{2} a^{2}-6.0 \sigma a^{4}+0.09 a^{6}-96.04=0
$$

From this equation we can get the data as in Table 1.

Noting that in the multivalue section in Table 1 there are several amplitudes for each value of $\sigma$ and it is consistent to theoretical results. The values in the blod correspond to the unstable state of the system as $\sigma$ increases and mutation occurs at the point $\sigma=0.75$.

Substituting $\left\{\sigma_{i}, a_{i}\right\}$ in Table 1 into Eq. (35), except the values in dark area, and solving them we get the values of $A, B, C$ and $D$ as follows

$$
\begin{gathered}
A=1.0387 \quad B=99.8408 \\
C=-5.9802 \quad D=0.0891
\end{gathered}
$$

Substituting them into Eq. (36), we obtain the physical parameters of the nonlinear system as follows

$$
\omega=4.996 n=0.102 \quad \beta=0.399
$$

These results of $\omega, n$ and $\beta$ are very close to the values of them we set above, thus the accuracy of the method presented in this paper is excellent. This conclusion can be explained by the principle of the method. From Section II we see our method is the combination of perturbation techniques and least square method which are effective methods 
Table 1

Frequency response data simulated by computer

\begin{tabular}{cccc}
\hline$\sigma$ & $\mathrm{a}$ & & \\
\hline-1 & 0.49394 & & \\
-0.5 & 0.93359 & & \\
-0.4 & 1.11467 & & \\
-0.3 & 1.35531 & & \\
-0.2 & 1.66498 & & \\
-0.1 & 2.03576 & & \\
0 & 2.44104 & & \\
0.1 & 2.85192 & & $\mathbf{1 . 4 0 9 2 2}$ \\
0.2 & 3.25015 & & $\mathbf{1 . 0 4 6 6 4}$ \\
0.3 & 3.62786 & & $\mathbf{0 . 8 5 2 0 8}$ \\
0.4 & 3.98285 & $\mathbf{2 . 9 6 9 4 5}$ & $\mathbf{0 . 7 2 2 9 8}$ \\
0.5 & 4.31488 & $\mathbf{3 . 6 9 0 4 8}$ & $\mathbf{0 . 6 7 2 7 9}$ \\
0.6 & 4.62311 & $\mathbf{4 . 2 3 0 9}$ & \\
0.7 & 4.90001 & $\mathbf{4 . 7 0 4 6 6}$ & \\
0.75 & 5.00000 & & \\
0.8 & & 0.62938 & \\
0.9 & & 0.55787 & \\
1 & & 0.50126 & \\
\hline
\end{tabular}

to deal with the weakly nonlinear vibration system and curve fitting, respectively, and the frequency response function is obtained from the first-order approximation of multiple scale method or other approximation methods, so that the accuracy of the method is as well as the one of first-order approximation of perturbation methods or the least square method. Meanwhile, the mathematical model, frequency response function of the weakly nonlinear system, of parameter identification of present method is a most important model describing the properties of weakly nonlinear systems. Above discussion shows that this method is a useful means for the parameter identification of weakly nonlinear vibration system.

Though the data $\left\{\sigma_{i}, a_{i}\right\}(i=1,2 \ldots, m)$ in the example is obtained by numerical simulation, we can achieve these data in practice. Noting that, in essence, the detuning parameter $\sigma$ is a small difference between the frequency of excitation and the linear natural frequency of the nonlinear system, thus, in practice test, we may change the frequency of excitation in a small range around the linear natural frequency, that is varying the detuning parameter $\sigma$. Recording down $\sigma$ and the corresponding amplitude $a$ of the system we obtain the test data $\left\{\sigma_{i}, a_{i}\right\}(i=1,2, \ldots, m)$. This means that the present method of parameter identification can be realized by experiment.

The study only involves one-degree-of -freedom nonlinear vibration system with cubic nonlinearity, but it is obvious that the proposed method of parameter identification can be applied to the system with higher nonlinearity or multi-degree-of-freedom nonlinear vibration systems.

\section{References}

[1] A.H. Nayfeh, Parametric identification of nonlinear systems, Computer and Structures 20 (1988), 487-493.

[2] J. Tang and Q. Huo, Identifying Parameters of Nonlinear System in Time Domain by the Response of Free Vibration, Journal of Vibration Engineering 2 (1989), 62-66, in Chinese.

[3] J. Tang and X. Li, Normal Perturbation Solution for Large Damped Autonomous Nonlinear Systems, Journal of Vibration and Shock 6(4) (1987).

[4] J. Tang, Phase plane method for the parameter identification of strongly nonlinear systems, Shock and Vibration 8 (2001), 49-53.

[5] K. Yasuda et al., Experimental identification technique of vibrating structures with geometrical nonlinear, ASME J. Appl. Mech. 64 (1997), $275-280$.

[6] K. Yasuda and K. Kamiya, Experimental identification technique of nonlinear beams in time domain, Nonlinear Dynamics 18 (1999), $185-202$.

[7] R. Bachmayer and L. Whitcomb, Adaptive parameter identification of an accurate nonlinear dynamical model for marine thrusters, $J$. Dynamic Systems, Measurement, and Control. 125 (2003), 489-491.

[8] R. P. Pacheco and V. Steffen Jr., Using orthogonal functions for identification and sensitivity analysis of mechanical systems, J Vibration and Control $\mathbf{8}$ (2002), 993-1021.

[9] R. Ghanem and F. Romeo, A wavelet-based approach for model and parameter identification of nonlinear systems, International J. Nonlinear Mechanics 36 (2001), 835-859. 
[10] A. Chatterjee and N. Vyas, Nonlinear parameter estimation using Volterra series with Multi-Tone excitation, XXIMAC Proceedings of the 20th International Model Analysis Conference, Los Angeles CA, 2002, pp. 880-885.

[11] D.Q. Nykamp and D.L. Ringach, Full identification of a linear-nonlinear system via cross-correlation analysis, Journal of Vision 2 (2002), $1-11$.

[12] H. Robert and K. László, Nonlinear System Identification - Input-Output Modeling Approach, Dordrecht: Kluwer Academic Publishers, 1999.

[13] A.H. Nayfeh and D.T. Mook, Nonlinear oscillations, Wiley: New York, 1979.

[14] A.H. Nayfeh, Introduction to perturbation techniques, Wiley: New York, 1988.

[15] W.T. Thomson, Theory of vibration with applications, New Jersey: Prentice-hall Inc., 1972.

[16] J. Deng, R. Ge and Z. Cheng, Calculation Method, Xi-an: Xi-an Jiaotong University Publishing Inc., 1985, in Chinese. 

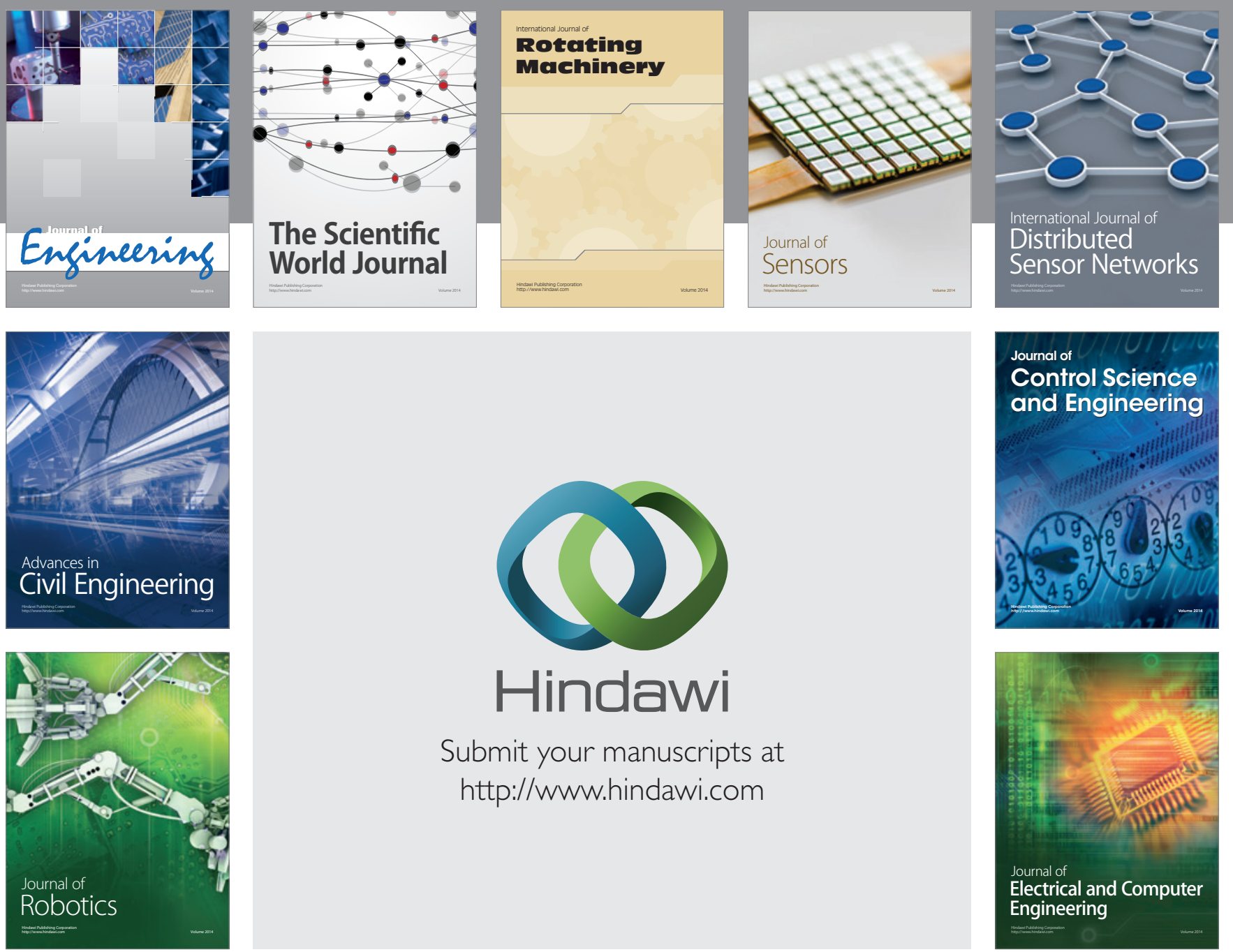

Submit your manuscripts at

http://www.hindawi.com
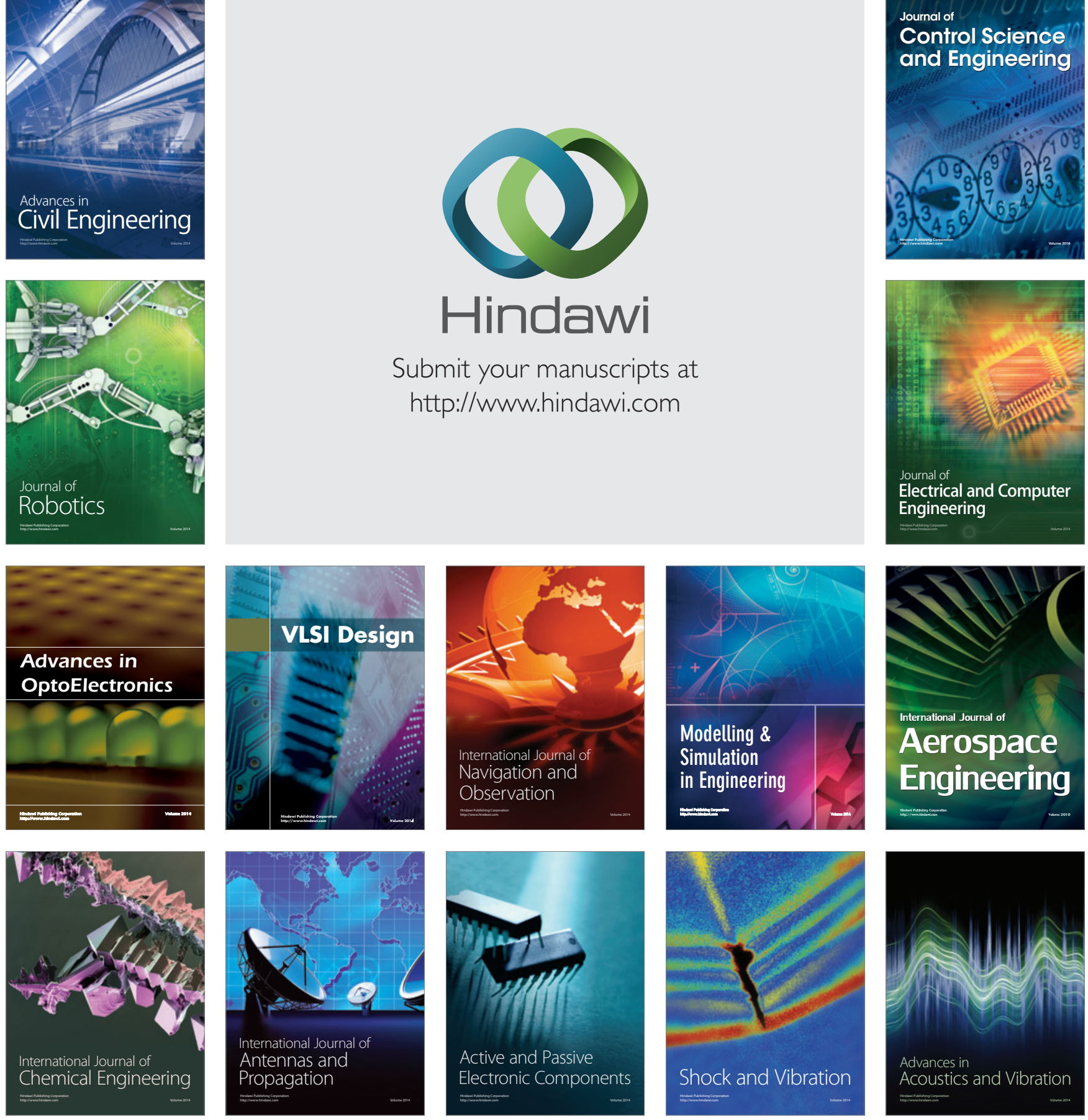